\title{
Ottimizzazione della terapia antiretrovirale in un piccolo paziente con infezione da HIV a trasmissione verticale: un case report. Optimizing antiretroviral therapy in an HIV infected child: a case report.
}

\section{Antonio Di Biagio}

Dipartimento di Scienze della Salute (DISSAL), Università degli Studi di Genova Ospedale Policlinico San Martino-IRCCS, Genova

\section{Introduzione}

L'ottimizzazione della terapia antiretrovirale rappresenta un passaggio fondamentale per tutte le persone con infezione da HIV (1).

A differenza degli anni passati, dove molto spesso la prima terapia era formata come minimo da due o tre compresse, oggi disponiamo già in prima linea terapeutica di regimi in singola compressa (STR). Questa situazione potrebbe determinare nel futuro un minor ricorso all'ottimizzazione delle terapie per riduzione del pill burden. Infatti, se un paziente inizia con una STR è difficile sostituire la terapia antiretrovirale per ridurre il numero di compresse. D’altro canto, però, a questo innovativo sviluppo, che ha riguardato indistintamente tutte le classi, analoghi non nucleosidici (NNRTI), inibitori della integrasi (INSTI) ed Inibitori della proteasi (PI), è seguito un altro fenomeno altrettanto repentino, ovvero l'introduzione nella pratica clinica di regimi a due farmaci: prima basati su un analogo nucleosidico della trascrittasi inversa (NRTI) ed un $\mathrm{PI}$, successivamente basati su un INSTI, in particolare dolutegravir.

Proprio queste strategie, basate su dolutegravir in associazione a lamivudina e dolutegravir in associazione a rilpivirina, offrono un'opportunità di riduzione del numero di compresse e del numero di farmaci utilizzati.

È evidente che, come tutte le strategie proposte, STR a tre farmaci piuttosto che dual (STR oppure a due compresse) devono essere valutate con il ragionamento clinico, devono insomma essere scelte durature, con l'obiettivo di migliorare la qualità della vita delle persone con infezione da HIV. Se tutto questo è vero per il paziente con infezione da HIV in età adulta, poche certezze si hanno nell'età neonatale, pediatrica e adolescenziale. Questa fascia di popolazione sconta diverse problematiche:

1) carenza di principi attivi,

2) scarsa disponibilità di formulazioni sciroppo,

3) scarsa palatabilità dei farmaci,

4) scarsa aderenza alle cure proposte (scuole, amici, non consapevolezza, etc). Inoltre, gli studi clinici delle sono carenti per numerosità e spesso propongono schemi di terapia già superati nel paziente adulto (periodi ON-OFF, monoterapia, etc). Certo è che la popolazione neonatale, pediatrica e adolescenziale nella sua globalità è una parte molto ristretta della casistica italiana e dei paesi ricchi. Ma alcuni casi purtroppo continuano a verificarsi anche alle nostre latitudini (2) e purtroppo sono sempre in numero inaccettabile nei paesi in via di sviluppo.

\section{Caso clinico}

Il caso clinico si riferisce ad un neonato, nato nel 2013, da un parto cesareo dopo 36 settimane di gestazione. Circa 24 ore dopo il parto, la madre è risultata positiva al test HIV. II test era stato fatto al travaglio, poiché la madre nei mesi prima e durante la gravidanza non lo aveva mai eseguito.

II piccolo ha ricevuto immediatamente una terapia antiretrovirale con zidovudina (AZT, alla dose di 2 mg per $\mathrm{Kg}$ di peso corporeo ogni 6 ore) e non è stato allattato al seno.
Autore

per la corrispondenza:

Antonio Di Biagio

Dipartimento di Scienze della Salute (DISSAL)

Università degli Studi di Genova

Ospedale Policlinico San Martino-IRCCS, Genova

Largo R. Benzi, 10 16132 Genoa, Italy

adibiagioa@gmail.com

Keywords:

HIV, ottimizzazione, terapia antiretrovirale

Potenziali conflitti di interesse:

nessuno.

JHA 2020; 5(1): 21-23

DOI: 10.19198/JHA31491 


\section{caso clinico}

A 48 ore dalla nascita, è stata quindi eseguita una determinazione dei livelli di HIV-RNA nel neonato che hanno evidenziato una HIV-RNA di oltre 10 milioni di copie per millilitro.

A 96 ore di età il neonato è stato trasferito in una Neonatologia con competenze sui bambini con HIV ed ha iniziato immediatamente una terapia antiretrovirale (ART) con AZT ( $8 \mathrm{mg} / \mathrm{kg}$ ogni 8 ore), lamivudina (3TC, $4 \mathrm{mg} / \mathrm{kg}$ due volte al giorno) e lopinavir/ritonavir (LPV/R, $16 \mathrm{mg} / \mathrm{kg}$ due volte al giorno).

HIV era un sottotipo $B$, il test di resistenza genotipica è stato eseguito e ha rivelato solo la presenza di V118I. I livelli di HIV-1 DNA associati alle cellule erano di 1750 copie/ $10^{6}$ PBMC.

A 120 ore dalla nascita la terapia ART è stata intensificata con l'aggiunta di raltegravir (RAL, $6 \mathrm{mg}$ / kg due volte al giorno) dopo aver ottenuto il parere dal comitato etico dell'Azienda Ospedaliera. A 8 giorni di età, il test di screening infantile per l'allele HLA-B 5701 è risultato negativo, quindi l'abacavir ( $A B C, 8 \mathrm{mg} / \mathrm{kg}$ due volte al giorno) ha sostituito I'AZT per prevenire la tossicità ematologica. La terapia ART è stata poi proseguita con ABC, 3TC e RAL. Non sono stati osservati segni di progressione clinica durante le prime otto settimane di terapia. (3) La carica virale del neonato è scesa $\mathrm{a}<200$ copie/ml dopo 6 mesi e a $<50$ copie/ml dopo 12 mesi di ART. A 18 mesi, mentre era sottoposto ad un regime basato su raltegravir $100 \mathrm{mg}$ BID, lamivudina 150 mg BID e abacavir 300 mg BID, ha perso gli anticorpi HIV materni (test non reattivo di quarta generazione HIV-1/2, Vironostika HIVAg/Ab, BioMérieux, e Western blot negativo).

Tale ART è stata portata avanti fino all'età di sei anni. Al raggiungimento del sesto anno di età, il superamento dei $25 \mathrm{Kg}$ e l'inizio della scuola elementare il regime terapeutico è stato modificato con lamivudina 300 QD e Tivicay 25 mg QD (foglietto illustrativo). Tale terapia è tollerata bene dal piccolo paziente senza effetti collaterali. HIVRNA è costantemente inferiore alle 50 copie/ml ed i linfociti CD4+ sono oltre 1000.

\section{Discussione}

Il caso clinico risulta di interesse per diverse ragioni. La prima considerazione e che lo scenario dove si svolge è il Nord industrializzato del nostro Paese e non un villaggio remoto dell'Africa. In Italia nel 2013 una mamma è arrivata al parto senza aver conoscenza del suo stato sierologico di HIV. In questo caso si tratta di una vera e propria missed opportunity, cioè aver perso l'occasione di far nascere il bambino senza infezione da HIV. Si sarebbe potuto somministrare la terapia alla mamma, eventualmente programmare un taglio cesareo e profilassi la madre ed il bambino durante il travaglio ed il post-partum.

La seconda nota è la gestione della terapia antiretrovirale nell'età neonatale pediatrica.

Le formulazioni sciroppo di abacavir (se HLAB5701 negativo) e lamivudina devono costituire l'asse portante, non è a mio avviso consentito in questa fascia di età l'impiego della zidovudina.

Il terzo farmaco della combinazione può essere sicuramente un INSTI, raltegravir per il suo profilo di tollerabilità e per la possibilità di differenziare il dosaggio può essere un valido sostituto della nevirapina.

Raltegravir nella formulazione da 100 mg in formulazione granulato per sospensione orale rappresenta un'ottima scelta in termini di tollerabilità (profilo lipidico, effetti gastro-intestinali) rispetto ai PI.

Se non si evidenziano mutazioni virali trasmesse, lo sciroppo di lopinavir/ritonavir per la sua scarsa palatabilità dovrebbe essere abbandonato, cosi come tutte le terapie alternative in questa fascia di età per promuovere l'impiego del raltegravir.

Infine, il precoce impiego di dolutegravir (appena età e/o peso lo consentono) e soprattutto la sua combinazione efficace con lamivudina in assenza di mutazioni è una scelta quasi obbligata per ridurre il pill burden.

Le motivazioni dell'ottimizzazione verso dolutegravir in associazione a lamivudina sono numerose: in primis le due piccole compresse, che a breve diventeranno una singola, sono molto facili da deglutire anche per il bambino; l'assunzione once-daily offre l'opportunità di non impegnare il piccolo con assunzioni durante le attività scolastiche e/o ludiche. L'assunzione serale, durante o dopo la cena, consente per esempio al mattino di andare a scuola senza dover assumere alcun tipo di terapia.

In conclusione, questo caso clinico dimostra la necessità di ottimizzare la terapia antiretrovirale come le pedine in una scacchiera. Ogni mossa deve essere pensata già con l'idea di cosa fare dopo. Ci auguriamo di seguire questo bimbo per molti anni, probabilmente arriveranno nuovi farmaci, forse l'eradicazione, quello che è certo che cercheremo di mantenere sempre la viremia non rilevata offrendogli la miglior terapia per ogni fase della sua vita. 


\section{caso clinico}

\section{REFERENCES}

1. Antinori A, Di Biagio A, Marcotullio S, et al. Evidence-based renewal of the Italian guidelines for the use of antiretroviral agents and the diagnostic-clinical management of HIV-1 infected persons. New Microbiol. 2018; 41:247-55.

2. Di Biagio A, Taramasso L, Gustinetti G, et al. Missed opportunities to prevent mother-to-child transmission of HIV in Italy. HIV Med. 2019; 20: 330-6.

3. Ripamonti D, Tatarelli P, Mangili G, et al. Potential role of raltegravir-based therapy to induce rapid viral decay in highly viraemic HIV-infected neonates. J Chemother Florence Italy. agosto 2016; 28: 337-40.

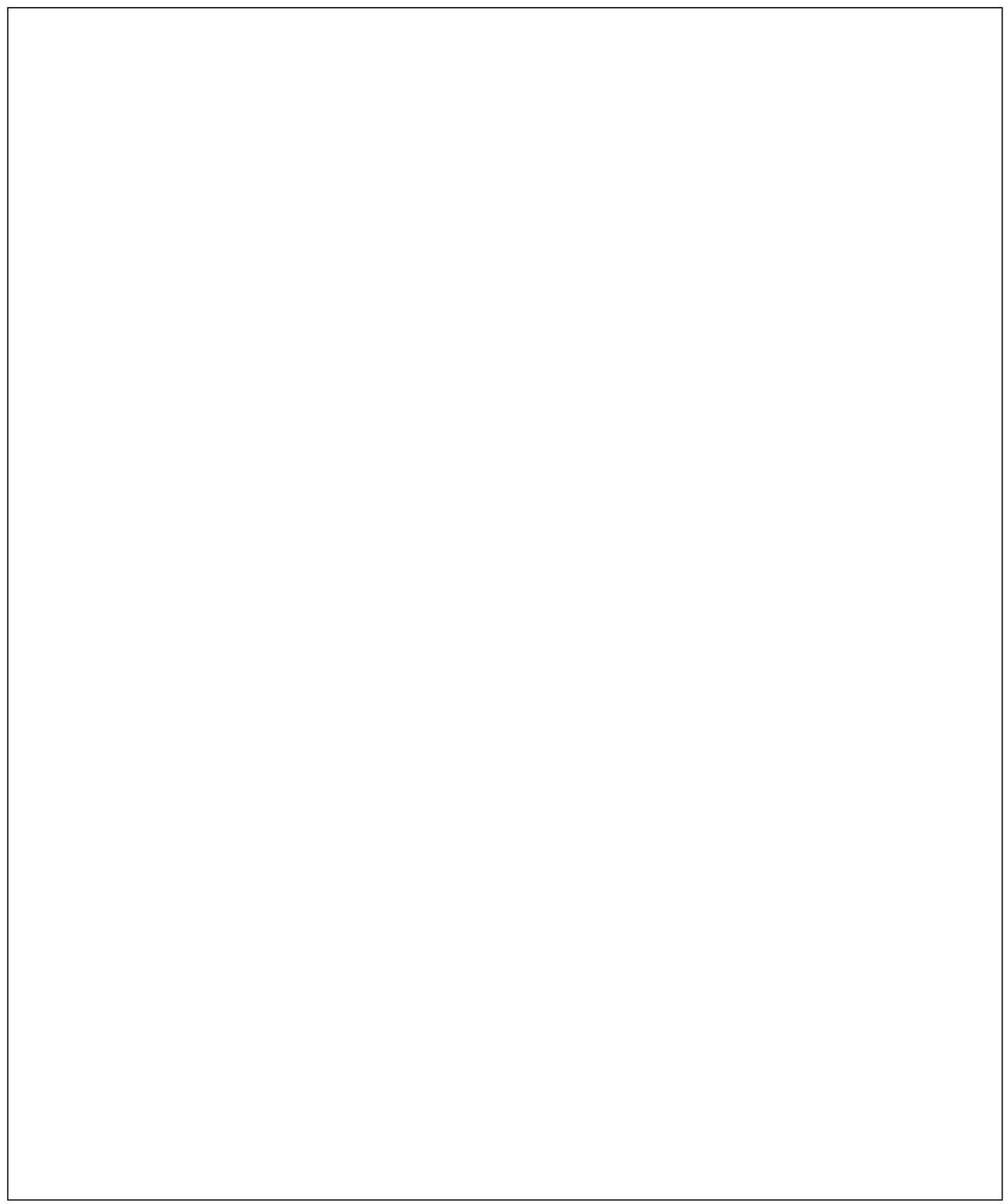

